\title{
ASYMPTOTIC INVERSE SERIES ${ }^{1}$
}

\section{VERNON A. KRAMER}

1. Introduction. For an operator $H(t)=H_{0}+t H_{1}+t^{2} H_{2}+\cdots$ we obtain the formal inverse expansion $H(t)^{-1}=A_{0}+t A_{1}+t^{2} A_{2}+\cdots$ where

$$
A_{n}=\sum_{p_{1}+\cdots+p_{r}-n}(-1)^{r} H_{0}^{-1} H_{p_{1}} H_{0}^{-1} H_{p_{2}} H_{0}^{-1} \cdots H_{p_{r}} H_{0}^{-1} .
$$

Under sufficiently stringent conditions on the operators $H_{i}$ this expansion will be valid; in particular, all elements $\phi$ will be in the domain of all $A_{i}$ and $\sum_{i} t^{i} A_{i} \phi$ will converge to $H(t)^{-1} \phi$. In more general situations an element $\phi$ may be in the domain of only the first few $A_{i}$. In this paper we obtain conditions (on $H_{i}$ and $\phi$ ) for the finite series $\sum_{i=0}^{N} t_{i} A_{i} \phi$ to be a valid asymptotic expansion of $H(t)^{-1} \phi$; that is, for $\lim _{t \rightarrow 0}\left\|t^{-N}\left\{H(t)^{-1} \phi-\sum_{t=0}^{N} t^{i} A_{i} \phi\right\}\right\|$ to be zero [Theorems 3.1 and 3.2]. Corresponding results are obtained for the inner product $\left(H(t)^{-1} \phi, \psi\right)$ [Theorems $4.1,4.2$, and 4.3 ].

The results of this study were obtained in the course of the solution of a problem of perturbation theory but they are of wider interest. Their application to perturbation theory will be given in a separate paper. The motivation of some of the hypotheses may be more evident from the point of view of that theory.

2. The Friedrichs extension. We are concerned with operators in a Hilbert space $\mathfrak{H C}$ with inner product $(\phi, \psi)$.

Definition 2.1. A symmetric operator $H$ is semi-bounded from below if there exists a constant $c$ such that $(H \phi, \phi) \geqq c(\phi, \phi)$ for all $\phi$ in $\mathfrak{D}(H)$, the domain of $H$.

For such operators, Friedrichs [1] has defined a self-adjoint extension with the same semi-bound. With no loss of generality we may assume the lower semibound to be one. Then $\mathbb{D}(H)$ with the bilinear form $(\phi, \psi)_{H}=(H \phi, \psi)$ has a completion which can be identified with a subspace $\mathfrak{D}^{\prime}$ of $\mathfrak{T}$. The Friedrichs extension of $H$ (denoted by $\widetilde{H}$ ) is defined as the restriction of the adjoint of $H$ to $D^{\prime}$. In this section we

Presented to the Society, November 12,1955; received by the editors June 17, 1955.

1 This paper is part of the author's doctoral dissertation submitted to the University of California, Berkeley, 1954, prepared under the guidance of Professor Frantisek Wolf. The research was partially supported by the Office of Ordnance Research, U. S. Army, Contract DA-04-200-ORD-171. 
give a few of the properties of this extension. For proofs, see Rellich [2].

Lemma 2.1. The subspace $D^{\prime}$ is the domain of the square root of $\tilde{H}$.

Definition 2.2. If $A$ and $B$ are symmetric operators, $A$ is greater than or equal to $B$ (in symbols, $A \geqq B$ ) if $D(A) \subseteq D(B)$ and $(A \phi, \phi)$ $\geqq(B \phi, \phi)$ for all $\phi$ in $D(A)$.

LEMMA 2.2. If $A \geqq B$, then $D\left(\tilde{A}^{1 / 2}\right) \subseteq \mathscr{D}\left(\tilde{B}^{1 / 2}\right)$ and $\left\|\tilde{A}^{1 / 2} \phi\right\| \geqq\left\|\tilde{B}^{1 / 2} \phi\right\|$ for all $\phi$ in $D\left(\tilde{A}^{1 / 2}\right)$.

LEMMA 2.3. If $A$ has a positive lower semi-bound $c$, then $\tilde{A}^{-1}$ exists and has norm not greater than $1 / c$.

LEMMA 2.4. If $A \geqq B \geqq c I$ with $c>0$, then $\widetilde{B}^{1 / 2} \tilde{A}^{-1 / 2}$ is bounded.

Caution. The hypotheses do not imply $\tilde{B} \tilde{A}^{-1}$ bounded. There is no simple relation between $D(\tilde{A})$ and $D(\tilde{B})$.

LEMMA 2.5. An element $\phi$ is in $D\left(\widetilde{H}^{1 / 2}\right)$ if and only if there exists a sequence $\left\{\phi^{\alpha}\right\}$ of elements of $D(H)$ such that

(1) $\lim _{\alpha} \phi^{\alpha}=\phi$,

(2) $\lim _{\alpha, \beta}\left(H\left(\phi^{\alpha}-\phi^{\beta}\right), \phi^{\alpha}-\phi^{\beta}\right)=0$.

This sequence has the further properties

(3) $\lim _{\alpha}\left(H \phi^{\alpha}, \phi^{\alpha}\right)=\left(\widetilde{H}^{1 / 2} \phi, \widetilde{H}^{1 / 2} \phi\right)$,

(4) $\lim _{\alpha} \widetilde{H}^{1 / 2} \phi^{\alpha}=\widetilde{H}^{1 / 2} \phi$,

(5) $\lim _{\alpha}\left(H \phi^{\alpha}, \omega\right)=\left(\tilde{H}^{1 / 2} \phi, \tilde{H}^{1 / 2} \omega\right)$ for all $\omega$ in $D\left(\tilde{H}^{1 / 2}\right)$.

The sequence $\left\{\phi^{\alpha}\right\}$ will be called an approximating sequence for $\phi$ with respect to $H$.

Now we impose once and for all certain basic assumptions on the operators $H_{i}$ of the series and redefine our problem. We assume $H_{0} \geqq I, H_{i} \geqq 0$ for $i>0$, and $D\left(H\left(t_{0}\right)\right)$ dense for some positive $t_{0}$. We restrict $t$ to the interval $0 \leqq t \leqq t_{0}$. Then $H(t) \geqq I$ for all $t$, and its Friedrichs extension $\tilde{H}(t)$ has an inverse with norm not greater than 1. We investigate $\widetilde{H}(t)^{-1}$ instead of $H(t)^{-1}$. We further assume that $H_{0}$ is the Friedrichs extension of its own contraction to $D\left(H\left(t_{0}\right)\right)$. Then $H_{0}$ is the Friedrichs extension of its contraction to $D(H(t))$ for all $t$. This last condition induces a type of continuity at $t=0$. If it were not satisfied with the given $H_{0}$, we would replace $H_{0}$ by the Friedrichs extension of its contraction to $D(H(t))$. With these assumptions it is easy to prove the lemmas below.

LEMMA 2.6. If $t_{1} \leqq t_{2}$, then $H\left(t_{1}\right) \leqq H\left(t_{2}\right)$. 
Lemma 2.7. For all $\phi$ in $D\left(\tilde{H}(t)^{1 / 2}\right),\|\phi\| \leqq\left\|\tilde{H}(t)^{1 / 2} \phi\right\|$.

LEMMA 2.8. The operator $H_{0}^{1 / 2} \tilde{H}(t)^{-1 / 2}$ is bounded, uniformly in $t$.

Let $R_{k}(t)$ denote the operator $H_{k}+t H_{k+1}+t^{2} H_{k+2}+\cdots$.

LEMMA 2.9. For all $\phi$ in $D\left(R_{1}(t)\right), t^{1 / 2}\left\|\tilde{R}_{1}(t)^{1 / 2} \phi\right\| \leqq\left\|\tilde{H}(t)^{1 / 2} \phi\right\|$.

This important inequality follows immediately from the obvious relation $t R_{1}(t) \leqq H(t)$.

3. The expansion of the inverse. In this section we give two inverse theorems. The first is of "pure asymptotic" type; that is, no error estimate is given. In the second theorem we make an additional assumption and obtain three results. The first can be interpreted as giving an error estimate for the previous theorem, the second is actually a lemma for use in the next section, and the third is again purely asymptotic. First we must prove a lemma.

Lemma 3.1. If $\phi$ and $\psi(t)$ belong to $D\left(\tilde{H}\left(t_{0}\right)^{1 / 2}\right)$ and $\phi$ belongs to $D\left(H_{0}\right)$, then $\left(\tilde{H}^{1 / 2} \phi, \tilde{H}^{1 / 2} \psi\right)=\left(H_{0} \phi, \psi\right)+t\left(\widetilde{R}_{1}^{1 / 2} \phi, \widetilde{R}_{1}^{1 / 2} \psi\right)$.

Proof. Note that $\psi$ may depend on $t$ but $\phi$ must be fixed. By Lemma 2.5 there exists a sequence $\left\{\phi^{\alpha}\right\}$ of elements of $D\left(\tilde{H}\left(t_{0}\right)\right)$ such that $\lim _{\alpha} \phi^{\alpha}=\phi$ and $\lim _{\alpha, \beta}\left(H\left(t_{0}\right)\left\{\phi^{\alpha}-\phi^{\beta}\right\}, \phi^{\alpha}-\phi^{\beta}\right)=0$. Expanding the second equation we obtain

$$
\lim _{\alpha, \beta}\left[\left(H_{0}\left\{\phi^{\alpha}-\phi^{\beta}\right\}, \phi^{\alpha}-\phi^{\beta}\right)+t_{0}\left(R_{1}\left(t_{0}\right)\left\{\phi^{\alpha}-\phi^{\beta}\right\}, \phi^{\alpha}-\phi^{\beta}\right)\right]=0 .
$$

Since both terms inside the bracket are positive

$$
\lim _{\alpha, \beta}\left(R_{1}\left(t_{0}\right)\left\{\phi^{\alpha}-\phi^{\beta}\right\}, \phi^{\alpha}-\phi^{\beta}\right)=0 .
$$

Thus $\phi$ is in $D\left(\widetilde{R}_{1}\left(t_{0}\right)^{1 / 2}\right)$ and hence in $\mathscr{D}\left(\widetilde{R}_{1}(t)^{1 / 2}\right)$ for all $t$. Furthermore, $\left\{\phi^{\alpha}\right\}$ is an approximating sequence for $\phi$ with respect to $R_{1}(t)$ for all $t$. A second application of Lemma 2.5 yields

$$
\begin{aligned}
\left(\widetilde{H}^{1 / 2} \phi, \tilde{H}^{1 / 2} \psi\right) & =\lim _{\alpha}\left(H(t) \phi^{\alpha}, \psi\right) \\
& =\lim _{\alpha}\left[\left(H_{0} \phi^{\alpha}, \psi\right)+t\left(R_{1} \phi^{\alpha}, \psi\right)\right] \\
& =\left(H_{0} \phi, \psi\right)+t\left(\tilde{R}_{1}^{1 / 2} \phi, \tilde{R}_{1}^{1 / 2} \psi\right) .
\end{aligned}
$$

Since the next theorem is the central result of the paper and its proof is rather complicated, some degree of motivation is in order. We must estimate the quantity $\left\|\tilde{H}(t)^{-1} \phi-\sum_{i=0}^{N} t^{i} A_{i} \phi\right\|$. If we were willing to impose sufficient domain conditions we could simply substitute this quantity for $x$ in the inequality $(x, x) \leqq(\tilde{H}(t) x, x)$. All terms of order less than $N$ in $\widetilde{H}(t) x$ would vanish, leaving a remainder 
$t^{N+1} R$. Then we would have $\|x\|^{2} \leqq t^{N+1}\|R\| \cdot\|x\|$ and from this the desired result. However, we do not wish to impose such heavy conditions; in particular, no domain conditions are to be imposed on the last term of the series, $A_{N} \phi$. So we approximate $A_{N} \phi$ by terms which do satisfy the domain conditions, but this approximation introduces two difficulties. First, the low order terms no longer strictly cancel. However, we can show that they "essentially" cancel in that their contribution can be made arbitrarily small, uniformly in $t$. The second problem is to prove that the remainder term originally due to $A_{N} \phi$ does not become too large when we pass to the approximation. The key to this proof is given by Lemma 2.9.

THEOREM 3.1. If $\phi$ satisfies the conditions (for fixed $N$ ),

(A) $\phi \in D\left(A_{i}\right)$ for $i \leqq N$;

(B) $A_{i} \phi \in \mathscr{D}(H(t))$ for $i<N$, then

$$
\lim _{t \rightarrow 0} t^{-N}\left\|\tilde{H}(t)^{-1} \phi-\sum_{i=0}^{N} t^{i} A_{i} \phi\right\|=0 .
$$

Proof. For $N=0$, the theorem reduces to the strong convergence of $\tilde{H}^{-1}$, a known result. Our proof assumes $N>0$, but a proof for $N=0$ could be obtained by making slight modifications.

Since $A_{N} \phi \in D\left(H_{0}\right)$ and $H_{0}$ is assumed to be the Friedrichs extension of its own contraction to $D(H(t))$, there exists a sequence $\left\{\phi^{\alpha}\right\}$ of elements of $D(H(t))$ such that $\lim _{\alpha} \phi^{\alpha}=A_{N} \phi$ and $\lim _{\alpha} H_{0}^{1 / 2} \phi^{\alpha}$ $=H_{0}{ }^{1 / 2} A_{N} \phi$. Using the first property we obtain

$$
t^{-N}\left\|\tilde{H}(t)^{-1} \phi-\sum_{i=0}^{N} t^{i} A_{i} \phi\right\|=\lim _{\alpha} t^{-N}\left\|\tilde{H}(t)^{-1}-\sum_{i=0}^{N-1} t^{i} A_{i} \phi-t^{N} \phi^{\alpha}\right\|
$$

uniformly in $t$. Let

$$
B^{\alpha}(t)=t^{-N}\left[\tilde{H}(t)^{-1} \phi-\sum_{i=0}^{N-1} t^{i} A_{i} \phi-t^{N} \phi^{\alpha}\right] .
$$

For fixed $\alpha$,

$$
\left\|B^{\alpha}(t)\right\| \leqq\left\|\tilde{H}(t)^{1 / 2} B^{\alpha}(t)\right\|
$$

By direct expansion we have

$$
\begin{aligned}
\left\|\tilde{H}(t)^{1 / 2} B^{\alpha}(t)\right\|^{2}= & t^{-N}\left(H(t)\left\{\tilde{H}(t)^{-1} \phi-\sum_{i=0}^{N-1} t^{i} A_{i} \phi\right\}, B^{\alpha}(t)\right) \\
& -\left(\tilde{H}(t)^{1 / 2} \phi^{\alpha}, \tilde{H}(t)^{1 / 2} B^{\alpha}(t)\right) .
\end{aligned}
$$

Applying hypotheses (B) to the first term on the right of (3.1) and Lemma 3.1 to the second we obtain 


$$
\begin{aligned}
\left\|\tilde{H}(t)^{1 / 2} B^{\alpha}(t)\right\|^{2} & =t^{-N}\left(\phi-\sum_{i=0}^{N-1} t^{i} \sum_{j=0}^{i} H_{j} A_{i-j} \phi-t^{N} \sum_{j=1}^{N} H_{j} A_{N-j}, B^{\alpha}(t)\right) \\
& -t \sum_{i=0}^{N-1}\left(R_{N+1-i} A_{i} \phi, B^{\alpha}(t)\right)-\left(H_{0} \phi^{\alpha}, B^{\alpha}(t)\right) \\
& -t\left(\tilde{R}_{1}(t)^{1 / 2} \phi^{\alpha}, \widetilde{R}_{1}(t)^{1 / 2} B^{\alpha}(t)\right) .
\end{aligned}
$$

The first term on the right reduces to $-\left(\sum_{j=1}^{N} H_{j} A_{N-j} \phi, B^{\alpha}(t)\right)$. Combining the first and third terms on the right of (3.2) we obtain

$$
\begin{aligned}
\left\|\tilde{H}(t)^{1 / 2} B^{\alpha}(t)\right\|^{2}= & -\left(H_{0}^{1 / 2}\left\{\phi^{\alpha}-\sum_{j=1}^{N} H_{0}^{-1} H_{j} A_{N-j} \phi\right\}, H_{0}^{1 / 2} B^{\alpha}(t)\right) \\
& -t \sum_{i=0}^{N-1}\left(R_{N+1-i}(t) A_{i} \phi, B^{\alpha}(t)\right) \\
& -t\left(\tilde{R}_{1}(t)^{1 / 2} \phi^{\alpha}, \tilde{R}_{1}(t)^{1 / 2} B^{\alpha}(t)\right) .
\end{aligned}
$$

By Lemmas 2.6, 2.7, and 2.9, $\left\|H_{0}^{1 / 2} B^{\alpha}(t)\right\|, t^{1 / 2}\left\|\widetilde{R}_{1}(t)^{1 / 2} B^{\alpha}(t)\right\|$, and $\left\|B^{\alpha}(t)\right\|$ are all less than or equal to $\left\|\tilde{H}(t)^{1 / 2} B^{\alpha}(t)\right\|$. Consequently

$$
\begin{aligned}
\left\|\widetilde{H}(t)^{1 / 2} B^{\alpha}(t)\right\| \leqq\left\|H_{0}^{1 / 2}\left\{\phi^{\alpha}-\sum_{j=1}^{N} H_{0}^{-1} H_{j} A_{N-j} \phi\right\}\right\| \\
+t\left\|\sum_{i=0}^{N-1} R_{N+1-i}(t) A_{i} \phi\right\|+t^{1 / 2}\left\|\tilde{R}_{1}(t)^{1 / 2} \phi^{\alpha}\right\| .
\end{aligned}
$$

From the second property of the sequence $\left\{\phi^{\alpha}\right\}$ it follows that

$$
\lim _{\alpha} H_{0}^{1 / 2}\left(\phi^{\alpha}-\sum_{j=1}^{N} H_{0}^{-1} H_{j} A_{N-j} \phi\right)=H_{0}^{-1 / 2}\left(-\sum_{j=0}^{N} H_{j} A_{N-j} \phi\right)=0 .
$$

For fixed $\alpha$, the last two terms of (3.3) tend to zero with $t$, so $\left\|\tilde{H}(t)^{1 / 2} B^{\alpha}(t)\right\|$ can be made arbitrarily small by proper choice of $\alpha$ and $t$.

For any positive $\delta$, there exists an $M$ such that for $\alpha \geqq M$ and $t \leqq t_{0}$,

$$
t^{-N}\left\|\tilde{H}(t)^{-1} \phi-\sum_{i=0}^{N} t^{i} A_{i} \phi\right\| \leqq\left\|\tilde{H}(t)^{1 / 2} B^{\alpha}(t)\right\|+\delta .
$$

Therefore, $t^{-N}\left\|\tilde{H}(t)^{-1} \phi-\sum_{i=0}^{N} t^{i} A_{i} \phi\right\|$ becomes arbitrarily small. This completes the proof of the theorem.

THEOREM 3.2. If $\phi$ satisfies the conditions (for fixed $N$ ),
(A) $\phi \in \mathscr{D}\left(A_{i}\right)$ for $i \leqq N$;
(B) $A_{2} \phi \in D(H(t))$ for $i<N$;
(C) $A_{N} \phi \in \mathscr{D}\left(\tilde{H}(t)^{1 / 2}\right)$, then 


$$
\begin{gathered}
\left\|\tilde{H}(t)^{-1} \phi-\sum_{i=0}^{N} t^{i} A_{i} \phi\right\| \leqq \\
t^{N+1}\left\|\sum_{i=0}^{N-1} R_{N+1-i}(t) A_{i} \phi\right\| \\
+t^{N+1 / 2}\left\|\tilde{R}_{1}(t)^{1 / 2} A_{N} \phi\right\|, \\
t^{-N}\left\|\tilde{R}_{1}(t)^{1 / 2}\left\{\tilde{H}(t)^{-1} \phi-\sum_{i=0}^{N} t^{i} A_{i} \phi\right\}\right\|
\end{gathered}
$$

is bounded, and

$$
\lim _{t \rightarrow 0} t^{(N+1 / 2)}\left\|\tilde{H}(t)^{-1} \phi-\sum_{i=0}^{N} t^{i} A_{i} \phi\right\|=0 .
$$

Proof. Let $C(t)=\tilde{H}(t)^{-1} \phi-\sum_{i=0}^{N} t^{i} A_{\imath} \phi$. By direct calculation

$$
\begin{aligned}
\left\|\widetilde{H}(t)^{1 / 2} C(t)\right\|^{2}= & \left(\tilde{H}(t)\left\{\tilde{H}(t)^{-1} \phi-\sum_{i=0}^{N-1} t^{i} A_{i} \phi\right\}, C(t)\right) \\
& -t^{N}\left(\tilde{H}(t)^{1 / 2} A_{N} \phi, \tilde{H}(t)^{1 / 2} C(t)\right) .
\end{aligned}
$$

Applying Lemma 3.1 to the last term of (3.4) and cancelling lower order terms we obtain

$$
\begin{aligned}
\left\|\tilde{H}(t)^{1 / 2} C(t)\right\|^{2}= & -t^{N+1}\left(\sum_{i=0}^{N-1} R_{N+1-i} A_{i} \phi, C(t)\right) \\
& -t^{N+1}\left(\tilde{R}_{1}(t)^{1 / 2} A_{N} \phi, \tilde{R}_{1}(t)^{1 / 2} C(t)\right) .
\end{aligned}
$$

From (3.5) and the fact that $\|C(t)\|$ and $t^{1 / 2}\left\|\widetilde{R}_{1}(t)^{1 / 2} C(t)\right\|$ are both less than or equal to $\left\|\tilde{H}(t)^{1 / 2} C(t)\right\|$, we conclude

$$
\begin{aligned}
\left\|\tilde{H}(t)^{1 / 2} C(t)\right\| \leqq & t^{N+1}\left\|\sum_{i=0}^{N-1} R_{N+1-i}(t) A_{i} \phi\right\| \\
& +t^{N+1 / 2}\left\|\tilde{R}_{1}(t)^{1 / 2} A_{N} \phi\right\| .
\end{aligned}
$$

The first and second conclusions of the theorem are immediate consequences of (3.6).

The third conclusion requires a more careful estimate of the last term of (3.5). Since $A_{N} \phi$ is in $D\left(\tilde{H}(t)^{1 / 2}\right)$ which contains $D\left(\widetilde{R}_{1}(t)^{1 / 2}\right)$, there exists an approximating sequence $\left\{\phi^{\alpha}\right\}$ for $A_{N} \phi$ with respect to $\widetilde{R}_{1}(t)^{1 / 2}$. Let $C^{\alpha}(t)=\tilde{H}(t)^{-1} \phi-\sum_{i=0}^{N-1} t^{i} A_{i} \phi-t^{N} \phi^{\alpha}$ and $D(t)$ $=\left\|\tilde{H}(t)^{1 / 2} C(t)\right\|$. Then for any $\delta>0$, there exists an $M$ such that for $\alpha>M$ and for all $t$

$$
\left|\left(\widetilde{R}_{1}(t)^{1 / 2} A_{N} \phi, \tilde{R}_{1}(t)^{1 / 2} C(t)\right)-\left(R_{1}(t) \phi^{\alpha}, C^{\alpha}(t)\right)\right| \leqq \delta t^{N} .
$$

Now from (3.5) we conclude 


$$
D(t)\left\{D(t)-t^{N+1} F(\alpha, t)\right\} \leqq \delta t^{2 N+1}
$$

where $F(\alpha, t)$ is bounded with respect to $t$ for fixed $\alpha$. We may assume the expression inside the braces is positive for sufficiently small $t$; otherwise, the desired result would follow immediately. Now one of the factors on the left must be less than $\delta^{1 / 2} t^{N+1 / 2}$. Either factor gives us the result that $D(t)$ tends to zero with $t^{N+1 / 2}$. Since $\| \tilde{H}(t)^{-1} \phi$ $-\sum_{i=0}^{N} t^{i} A_{i} \phi \| \leqq D(t)$, the last conclusion follows.

This concludes the proof of the theorem.

4. The expansion of the inner product $\left(\tilde{H}(t)^{-1} \phi, \psi\right)$. The basic idea of this section is to have $\phi$ and $\psi$ share the domain requirements for an expansion to be valid. We adopt the notation $o\left(t^{N}\right)$ and $O\left(t^{N}\right)$ to represent numbers or vectors whose product with $t^{-N}$ tends to zero or remains bounded respectively as $t$ tends to zero.

TheORem 4.1. If $\phi$ and $\psi$ satisfy the conditions of Theorem 3.1 for order $N$ and $M$ respectively, then

$$
\begin{aligned}
\left(\tilde{H}(t)^{-1} \phi, \psi\right)= & \sum_{i=0}^{M-1} t^{i}\left(\phi, A_{\imath} \psi\right) \\
& -\sum_{j=M}^{M+N} t^{i} \sum_{k=M}^{j} \sum_{p=0}^{M-1}\left(A_{j-k} \phi, B_{k-p} A_{p} \psi\right)+o\left(t^{M+N}\right) .
\end{aligned}
$$

Proof. The theorem is trivial for $M=0$, so we suppose $M>0$. Since $\tilde{H}(t)^{-1} \phi$ must belong to $D(\tilde{H}(t))$, there exists a sequence $\left\{\xi^{\alpha}(t)\right\}$ of elements of $D(H(t))$ for each $t$ such that

(1) $\lim _{\alpha} \xi^{\alpha}(t)=\tilde{H}(t)^{-1} \phi$,

(2) $\lim _{\alpha}\left(H(t) \xi^{\alpha}(t), \omega\right)=(\phi, \omega)$ for all $\omega$ in $D\left(\tilde{H}(t)^{1 / 2}\right)$.

For $i<M, A_{i} \psi$ is in $D(H(t))$ so that

$$
\lim _{\alpha}\left(H(t) \xi^{\alpha}(t), \sum_{i=0}^{M-1} t^{i} A_{i} \psi\right)=\left(\phi, \sum_{i=0}^{M-1} t^{i} A_{i} \psi\right) .
$$

On the other hand, we obtain by direct calculation

$$
\left(H(t) \xi^{\alpha}(t), \sum_{i=0}^{M-1} t^{i} A_{i} \psi\right)=\left(\xi^{\alpha}(t), \psi\right)+\sum_{k \geqq M} t^{k} \sum_{p=0}^{M-1}\left(\xi^{\alpha}(t), H_{k-p} A_{p} \psi\right)
$$

Taking the limit of (4.2) with respect to $\alpha$ and comparing with (4.1), we have

$$
\left(\tilde{H}(t)^{-1} \phi, \psi\right)=\left(\phi, \sum_{i=0}^{M-1} t^{i} A_{i} \psi\right)-\sum_{k \geqq M} t^{k} \sum_{p=00}^{M-1}\left(\tilde{H}(t)^{-1} \phi, H_{k-p} A_{p} \psi\right) .
$$

Expanding $\tilde{H}(t)^{-1} \phi$ to order $N$ on the right and collecting terms we obtain the desired expansion. 
For simplicity, we state our next theorem for operators of the form $H_{0}+t H_{1}$. In this case $A_{i}=(-1)^{i} H_{0}^{-1}\left(H_{1} H_{0}^{-1}\right)^{i}$.

Theorem 4.2. If $H(t)=H_{0}+t H_{1}$ and $A_{N} \phi$ and $A_{M} \psi$ are defined and belong to $D\left(\tilde{H}(t)^{1 / 2}\right)$, then

$$
\begin{aligned}
\left(\tilde{H}(t)^{-1} \phi, \psi\right)= & \sum_{i=0}^{M} t^{i}\left(\phi, A_{i} \psi\right)-\sum_{j=0}^{N-1} t^{M+j+1}\left(\left(H_{1} H_{0}^{-1}\right)^{j+1} \phi, A_{M} \psi\right) \\
& +t^{M+N+1}\left(H_{1}^{1 / 2} A_{N} \phi, H_{1}^{1 / 2} A_{M} \psi\right)+o\left(t^{M+N+1}\right) .
\end{aligned}
$$

Proof. This theorem is nontrivial for $N$ or $M$ zero and we do not assume them different from zero. Since $\tilde{H}(t)^{-1} \phi \in D\left(\widetilde{H}(t)^{1 / 2}\right) \subseteq D\left(\widetilde{H}_{1}^{1 / 2}\right)$, there exists a sequence $\left\{\xi^{\alpha}(t)\right\}$ of elements of $\mathscr{D}(H(t))$ such that

(1) $\lim _{\alpha} \xi^{\alpha}(t)=\tilde{H}(t)^{-1} \phi$,

(2) $\lim _{\alpha}\left(H(t) \xi^{\alpha}(t), \chi\right)=(\phi, \chi)$ for all $\chi$ in $D\left(\tilde{H}(t)^{1 / 2}\right)$,

and

(3) $\lim _{\alpha}\left(H_{1} \xi^{\alpha}(t), \omega\right)=\left(\tilde{H}_{1}^{1 / 2} \tilde{H}(t)^{-1} \phi, \tilde{H}_{1}^{1 / 2} \omega\right)$ for all $\omega$ in $D\left(\tilde{H}_{1}^{1 / 2}\right)$.

From property (2) it follows that

$$
\lim _{\alpha}\left(H(t) \xi^{\alpha}(t), \sum_{i=0}^{M} t^{i} A_{i} \psi\right)=\left(\phi, \sum_{i=0}^{M} t^{i} A_{i} \psi\right)
$$

By direct calculation we obtain

$$
\left(H(t) \xi^{\alpha}(t), \sum_{i=0}^{M} t^{i} A_{i} \psi\right)=\left(\xi^{\alpha}(t), \psi\right)-t^{M+1}\left(\tilde{H}_{1}^{1 / 2} \xi^{\alpha}(t), \tilde{H}_{1}^{1 / 2} A_{M} \psi\right)
$$

From (4.3) and (4.4) we have

$$
\left(\tilde{H}(t)^{-1} \phi, \psi\right)=\sum_{i=0}^{M} t^{i}\left(\phi, A_{i} \psi\right)-t^{M+1}\left(\tilde{H}_{1}^{1 / 2} \tilde{H}(t)^{1 / 2} \phi, \tilde{H}_{1}^{1 / 2} A_{M} \psi\right) .
$$

By the second conclusion of Theorem 3.2, $\tilde{H}(t)^{-1} \phi=\sum_{i=0}^{N} t^{i} A_{i} \phi$ $+t^{N} \rho(t)$ where $\rho(t)$ tends to zero with $t$ and $\widetilde{H}_{1}^{1 / 2} \rho(t)$ is bounded. Therefore,

$$
\begin{aligned}
\left(\tilde{H}_{1}^{1 / 2} \tilde{H}(t)^{-1} \phi, \tilde{H}_{1}^{1 / 2} A_{M} \psi\right)= & \sum_{i=0}^{N} t^{i}\left(\tilde{H}_{1}^{1 / 2} A_{i} \phi, \tilde{H}_{1}^{1 / 2} A_{M} \psi\right) \\
& +t^{N}\left(\tilde{H}_{1}^{1 / 2} \rho(t), \tilde{H}_{1}^{1 / 2} A_{M} \psi\right)
\end{aligned}
$$

Since $\tilde{H}_{1}^{1 / 2} \rho(t)$ is bounded, there exists an approximating sequence 
such that $\left(\widetilde{H}_{1}^{1 / 2} \rho(t), \tilde{H}_{1}^{1 / 2} A_{M} \psi\right)=\lim _{\beta}\left(\rho(t), H_{1} \omega^{\beta}\right)$ uniformly in $t$. For each fixed $\beta \lim _{t \rightarrow 0}\left(\rho(t), H_{1} \omega^{\beta}\right)=0$. Interchanging limits we have

$$
\lim _{t \rightarrow 0}\left(\tilde{H}_{1}^{1 / 2} \rho(t), \tilde{H}_{1}^{1 / 2} A_{M} \psi\right)=0 .
$$

The theorem follows from (4.5), (4.6), and (4.7).

Theorem 4.2 can be generalized to operators with more than two terms, but the result is rather awkward and difficult to state. In the special case of $N=M=0$ we do obtain a concise and useful theorem.

TheOREM 4.3. If $H(t)=H_{0}+t H_{1}+t^{2} H_{2}+\cdots$ and $H_{0}^{-1} \phi$ and $H_{0}^{-1} \psi$ belong to $\mathcal{D}\left(\tilde{H}(t)^{1 / 2}\right)$, then

$$
\left(\tilde{H}(t)^{-1} \phi, \psi\right)=\left(H_{0}^{-1} \phi, \psi\right)-t\left(\tilde{H}_{1}^{1 / 2} H_{0}^{-1} \phi, \tilde{H}_{1}^{1 / 2} H_{0}^{-1} \psi\right)+o(t) .
$$

Proof. The proof involves no new ideas and will be omitted.

We give a simple example for which neither hypotheses nor conclusion of Theorem 4.3 hold. Consider in $L^{2}(-\infty,+\infty)$ the operator

$$
H(t)=H_{0}+t^{2} H_{2}
$$

where

$$
\begin{gathered}
H_{0} f(x)=-\phi(x) \int_{-\infty}^{\infty} f(\xi) \phi(\xi) d \xi-2 f(x), \\
H_{2} f(x)=x^{2} f(x)
\end{gathered}
$$

and

$$
\phi(x)=\left\{\pi\left(1-x^{2}\right)\right\}^{-1 / 2} .
$$

Then $H(t)$ is self-adjoint and $H_{0}$ and $H_{2}$ satisfy our fundamental assumptions. Obviously, $H^{-1} \phi=\phi$ and $\phi$ does not belong to $D\left(\tilde{H}(t)^{1 / 2}\right)$ since it is not in $\mathcal{D}\left(H_{2}^{1 / 2}\right)$. Thus the hypotheses of Theorem 4.3 are not satisfied. Since $H_{1}=0$, the conclusion of the theorem would be $\left(H(t)^{-1} \phi, \phi\right)=\left(H_{0}^{-1} \phi, \phi\right)+o(t)$, but by direct calculation we obtain $\left(H(t)^{-1} \phi, \phi\right)=\left(H_{0}^{-1} \phi, \phi\right)+2^{1 / 2} t+o(t)$.

\section{BIBLIOGRAPHY}

1. K. O. Friedrichs, Spektraltheorie halbbeschränkter Operatoren und Anwendung auf die Spektralzerlegung von Differentialoperatoren, Math. Ann. vol. 109 (1934) pp. 465-487.

2. F. Rellich, Halbbeschränkte gewohnliche Differentialoperatoren zweiter Ordnung, Math. Ann. vol. 122 (1951) pp. 343-368.

University of California, Berkeley 